\title{
Three-year efficacy and patency follow-up of decellularized human internal mammary artery as a novel vascular graft in animal models
}

Abdol-Mohammad Kajbafzadeh, MD, ${ }^{\text {a }}$ Reza Khorramirouz, MD, ${ }^{a}$ Seyedeh Maryam Kameli, MS, ${ }^{a}$ Kiarad Fendereski, ${ }^{a}$ Seyedeh Sima Daryabari, ${ }^{a}$ Seyed Mohammad Tavangar, MD, ${ }^{\mathrm{b}}$ and Bahram Azizi Garajegayeh, BA

\section{ABSTRACT}

Background: Various investigations have reported that the internal mammary artery (IMA) is an efficient and functional choice of conduit for vascular graft surgeries, especially for coronary artery bypass grafts; however, the quest to find an ideal vascular substitute remains. We hypothesized that acellular IMA could be an appropriate graft for small-diameter vascular bypasses that could be used in various surgeries including coronary artery bypass grafting.

Methods: We decellularized human IMAs and performed histologic evaluations and scanning electron microscopy to confirm the decellularization process and the preservation of the extracellular matrix. Subsequently, we grafted the scaffolds into the superficial femoral arteries of $8 \mathrm{New}$ Zealand rabbits with an end-toend anastomosis. Computed tomography angiograms were provided at 3, 12, and 36 months postoperatively. Subsequently, the animals were killed, and biopsies were taken for histologic and immunohistochemical assessments.

Results: Evaluation of the acellular tissue confirmed the efficacy of the decellularization protocol and the preservation of the extracellular matrix. All 8 animals survived the entire follow-up period. Doppler ultrasonography and computed tomography angiographies verified the conduit's patency. Histologic assessments depicted the recellularization of all 3 layers of the scaffold. Smooth muscle cells were detected in tunica media. Immunohistochemical assessments confirmed these findings.

Conclusions: In conclusion, we demonstrated that acellular human IMA could be used as an efficient small-diameter vascular substitute with high patency. These findings could pave the path for future investigations on the clinical application of acellular IMA as a novel vascular graft for small-diameter bypass surgeries. (J Thorac Cardiovasc Surg 2019;157:1494-502)

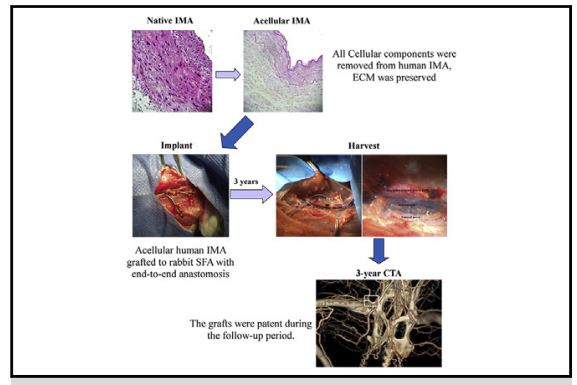

Acellular IMAs were grafted to the rabbit's SFA. The conduits stayed patent after 3 years.

\section{Central Message}

In this study, we implanted acellular human IMAs as novel vascular grafts in animal models. The findings indicated that this scaffold could provide us with an extended source of vascular substitutes.

\section{Perspective}

The grafted conduits stayed patent after 3 years of follow-up. Complete recellularization of all vascular layers was observed, indicative for replacement of the graft by the host tissue. Overall, no significant difference was detectable between the implanted scaffold and the animal's native artery after the follow-up period.

See Commentary page 1503 .
Cardiovascular diseases (CVDs) have the greatest global rates of morbidity and mortality. A common pathogenic

From the apediatric Urology and Regenerative Medicine Research Center, Children's Medical Center, ${ }^{\mathrm{b}}$ Department of Pathology, Shariati Hospital, and ${ }^{\mathrm{c}}$ Imaging Center, Children's Medical Center, Tehran University of Medical Sciences, Tehran, Iran.

This study was funded by Tehran University of Medical Sciences, grant number: 9501-84-30441.

Received for publication May 27, 2018; revisions received Aug 9, 2018; accepted for publication Aug 28, 2018; available ahead of print Nov 2, 2018.

Address for reprints: Abdol-Mohammad Kajbafzadeh, MD, No. 62, Dr. Gharib's St, Keshavarz Boulevard, Tehran 1419433151, Iran (IRI) (E-mail: kajbafzd@sina. tums.ac.ir).

$0022-5223 / \$ 36.00$

Copyright (c) 2018 by The American Association for Thoracic Surgery

https://doi.org/10.1016/j.jtcvs.2018.08.106 pathway of CVDs includes generation and maturation of atherosclerotic plaques within the coronary arteries, which could lead to arterial constricting, embolism, and subsequent myocardial ischemic injuries. ${ }^{1,2}$ Although the application of percutaneous coronary intervention has rapidly advanced over the recent years, coronary artery bypass graft (CABG) surgery is still the gold standard

- Scanning this $\mathrm{QR}$ code will take you to the article title page to access supplementary information.

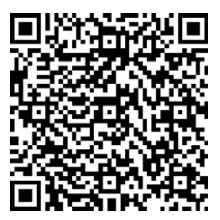



Abbreviations and Acronyms
$\alpha$-SMA $=$ alpha smooth muscle actin
$\mathrm{CABG}=$ coronary artery bypass graft
$\mathrm{CT}=$ computed tomography
$\mathrm{CVD}=$ cardiovascular disease
$\mathrm{ECM}=$ extracellular matrix
$\mathrm{H} \& \mathrm{E}=$ hematoxylin and eosin
IMA = internal mammary artery
PBS = phosphate-buffered saline
SDS $=$ sodium dodecyl sulfate
SEM = scanning electron microscopy

treatment for various CVDs, such as multivessel or left main stem coronary artery diseases. ${ }^{3}$ Currently, long saphenous vein is the most common conduit exercised in CABG surgeries because of its simple accessibility; however, saphenous vein graft failure could be a limitation associated with arterial occlusion and adverse cardiac effects. ${ }^{4,5}$ Long-term follow-up investigations revealed that application of venous conduits for $\mathrm{CABG}$ surgeries is accompanied by $50 \%$ chance of occlusion within 10 to 15 years after the procedure. $^{6}$

In 1986, Loop and colleagues ${ }^{7}$ demonstrated the advantages of application internal mammary artery (IMA) grafts versus vein grafts. The patients with arterial graft showed a significantly greater survival rate after 10 years of followup. Moreover, various investigations have reported that the application of unilateral or bilateral IMA could increase the efficiency rate and the functional properties in comparison with other arterial or venous conduits; however, the challenge to find an ideal vascular substitute remains. ${ }^{8}$

Approximately $30 \%$ of patients do not retain viable vessels for CABG vascular reconstruction, often due to a systemic vascular disease. ${ }^{9}$ In addition, a repeated surgery is required in various cases. Thus, tissue-engineered vascular grafts are being investigated as potential alternatives for vascular-reconstruction procedures. ${ }^{10}$ Weinberg and Bell introduced the first engineered conduit in 1986. Although they provided proof of concept for the application of engineered tissues as vascular conduits, the grafts were unable to maintain the physiologic pressure for more than 6 weeks. ${ }^{11}$

Since that time, expanded polytetrafluoroethylene and polyethylene terephthalate (Dacron) have become broadly used in the production of synthetic vascular substitutes. Nevertheless, they are inapplicable for small-sized arteries. Also, the risk of thrombus formation, compliance mismatch, and the absence of endothelial cells, which results in low patency rate of these grafts, have hampered the application of these synthetic materials. ${ }^{12}$

In the past decade, investigations have revealed that decellularization of various tissues and organs using the appropriate methods could produce cell-free scaffolds with maintained extracellular matrix (ECM) components (eg, collagen, elastin, and fibronectin), structure, and properties. ${ }^{13}$ Several studies have demonstrated that the decellularization of allogeneic or xenogeneic vascular tissues could provide a reliable source of conduits with appropriate properties. Application of natural scaffolds such as rat aorta, porcine aorta, and porcine iliac vessels for vascular regeneration has already resulted in considerable outcomes. ${ }^{14,15}$ Furthermore, human-engineered tissues grafted into baboon models showed a patency rate of $88 \%{ }^{16}$

Recently, we demonstrated successful decellularization of human IMA by a Triton X-100 and sodium dodecyl sulfate (SDS) protocol. Evaluation of the decellularized tissue confirmed the preservation of microarchitecture and the similarity of mechanical properties to the native tissue. ${ }^{17}$ In a subsequent study, our research team used rat omentum as a natural bioreactor for in vivo recellularization of acellular human IMAs. The outcomes were promising concerning recellularization process, angiogenesis, and lumen patency. ${ }^{18}$

In this study, we decellularized and characterized human IMAs based on our previously described protocol and subsequently grafted the acellular conduits into rabbit femoral artery as a small-sized model. We hypothesized that this acellular graft could be used in small-diameter bypass surgeries.

\section{MATERIALS AND METHODS \\ Acellular Scaffold Preparation}

The study protocol was reviewed and overseen by The Tehran University of Medical Sciences Ethics Committee. Substitute decision-makers of the patients declared brain-dead provided written, informed consent for the use of tissues for experimental studies.

Twenty samples of human IMA were harvested from its origin in the subclavian artery to the point of its bifurcation. These samples were collected from patients declared brain-dead (men younger than 50 years) during heart transplantation surgery. The samples were proximally cannulated using a 14-gauge catheter and were preserved in a phosphate-buffered saline (PBS) solution the decellularization process.

The decellularization process was performed according to the perfusion-based Triton X-100 and SDS protocol, as we described in our previous studies. ${ }^{17,18}$ To summarize in brief, the tissues are perfused with $1 \%$ solution of Triton X-100 (Sigma-Aldrich, St Louis, Mo) for 24 hours at the flow rate of $60 \mathrm{~mL} / \mathrm{min}$ and subsequently perfused with $1 \%$ solution of SDS (Sigma-Aldrich) for another 24 hours at the same flow rate. The grafts are eventually preserved in a PBS solution containing penicillin, amphotericin, and gentamicin at $4{ }^{\circ} \mathrm{C}$ temperature for further evaluations and implantation into animal models.

\section{Scanning Electron Microscopy (SEM)}

SEM assessments were carried out to confirm the efficacy of the decellularization process and preservation of the conduit's ECM. We preserved the samples in a $2.5 \%$ glutaraldehyde at $4{ }^{\circ} \mathrm{C}$ for 24 hours and washed them with PBS for 3 times (30 minutes each). Subsequently, they were dehydrated by graded series of ethanol $(30 \%, 50 \%, 70 \%, 90 \%$, and $100 \%$ ) and 15 minutes of preservation under a critical point dryer (Autosamdri814; Tousimis Research Corp, Rockville, Md). Finally, all of the samples 
were coated with gold (approximately $3 \mathrm{~nm}$ of thickness) and were visualized by SEM (S3500 N; Hitachi High Technologies America, Schaumburg, III) at the voltage of $10 \mathrm{kV}$ and a working distance of $8 \mathrm{~mm}$.

\section{Surgical Techniques}

All the animal procedures were approved by The Animal Ethics Committee of the Tehran University of Medical Sciences, School of Medicine and Education Section of Basic Sciences and were executed in accordance with the Animal Welfare Act and the Guide for the Care and Use of Laboratory Animals. Subsequent to preparation of the acellular scaffolds, we implanted the conduits into femoral artery of rabbits.

\section{Rabbit Femoral Artery}

Eight adult female New Zealand rabbits (2500-3000 g of body weight) were anesthetized by intramuscular injection of ketamine $(35 \mathrm{mg} / \mathrm{kg})$ and xylazine $(5 \mathrm{mg} / \mathrm{kg})$. We shaved the right inguinal area and made an incision to expose the femoral artery, vein, and nerve. We clamped the superficial femoral artery below the origins of profunda femoris artery and superficial femoral artery, in order to preserve the blood supply of limb and prevent possible ischemia. Afterwards, the artery was transected and end-to-end anastomoses were performed between the IMA scaffold and the superficial femoral artery distal to the bifurcation point using interrupted PROLENE
9-0 sutures (Ethicon, Somerville, NJ). Finally, the clamp was released and the patency of the graft was evaluated. The muscular layer was closed by continuous 5-0 nonabsorbable sutures. We evaluated the patency of the implanted conduits by Doppler ultrasonography 2 weeks and a month after the surgery (Figure 1 and Video 1).

\section{Histologic and Immunohistochemical Evaluations}

Biopsies were taken from native and acellular IMAs to evaluate the decellularization process and preservation of the ECM. Also, the animals were killed after 3 years of follow-up and the grafts were harvested for histologic and immunohistochemical evaluations. The native superficial femoral arteries were collected to be evaluated as a control group.

All the specimens were fixed in $10 \%$ (wt/vol) natural buffered formalin (Merck, Darmstadt, Germany) for 24 hours ( $\mathrm{pH} 7.4$ ) in room temperature, washed in distilled water, dehydrated in graded series of ethanol, and embedded in paraffin wax. Each sample was sectioned into 5- $\mu \mathrm{m}$ thicknesses for further evaluations. The samples were rehydrated and stained with hematoxylin and eosin (H\&E). Picrosirius red and Russell-Movat's pentachrome staining were carried out for native and acellular IMAs as previously described. ${ }^{18}$ Picrosirius red staining was applied for collagen typing under polarized light (type-I collagen: yellow-orange birefringence; type-III collagen: green birefringence). Furthermore, Pentachrome staining
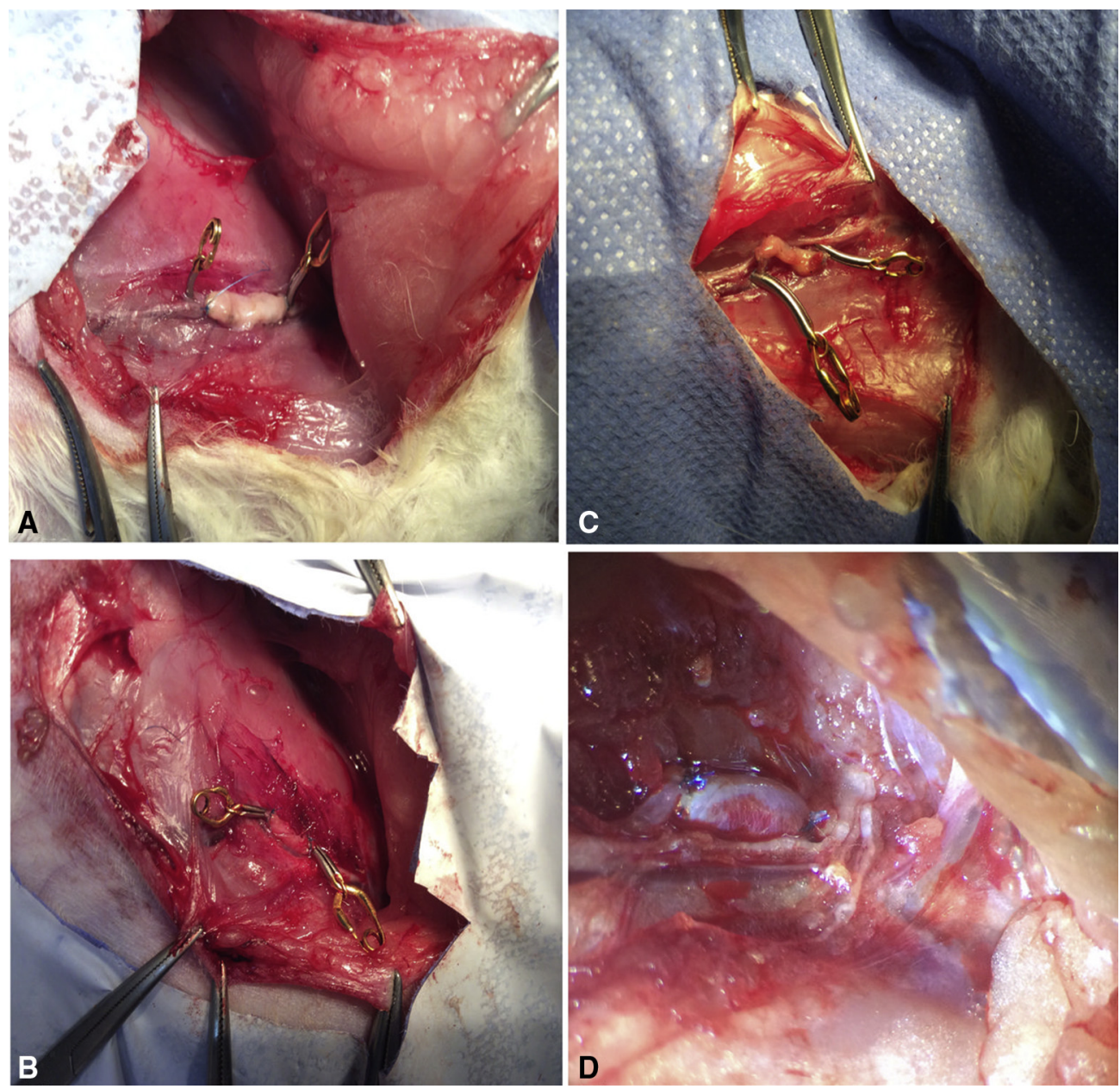

FIGURE 1. Surgical procedures. A-D, The scaffolds are grafted to rabbit superficial femoral artery below the bifurcation point with an end-to-end anastomosis. 


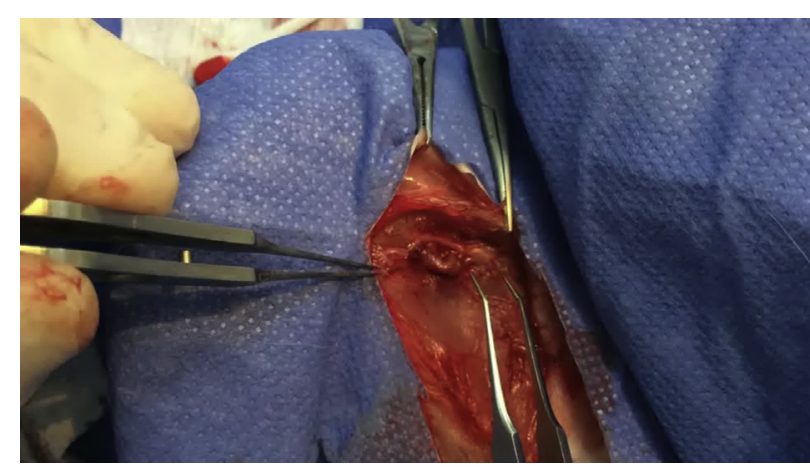

VIDEO 1. An anastomosed internal mammary artery graft on the origin of superficial femoral artery is shown. The clamps are released, and blood flow could be observed without any leakage. The implanted conduit is pulsatile, which is indicative for patency of the lumen. Video available at: https:// www.jtcvs.org/article/S0022-5223(18)32501-7/fulltext.

was used to differentiate connective tissue components. The grafted conduits and native superficial arteries were explanted after 3 years of follow-up and the samples were stained with H\&E and Masson's trichrome. Also, we performed the immunohistochemical evaluations for these samples by anti-alpha smooth muscle actin ( $\alpha$-SMA, ab5694; Abcam, Cambridge, United Kingdom), anti-CD31 (ab28364; Abcam), and anti-CD34 (ab185732; Abcam) antibodies. The $\alpha$-SMA staining was carried out to determine the quality of tunica media. The immunohistochemical assessments of CD31 and CD34 were to delineate the regeneration of endothelial layer in the implanted tissue.

An expert pathologist evaluated the specimens in a double-blinded condition. The images were taken by a Nikon camera DXM 1200. Photoshop 10.0 software (Adobe Systems, Inc, Mountain View, Calif) and Image Pro (Image Pro Inc, Boston, Mass) were used to analyze the images.

\section{Computed Tomography (CT) Angiography}

Before CT angiography assessments, a pulse oximeter (Bruker, Billerica, Mass) was used to evaluate oxygen saturation and blood supply of both lower limbs in all animals. We performed CT angiographies (GE BrightSpeed 16; GE Healthcare, Chicago, Ill) to evaluate the patency of the grafted tissues 3 months, 1 year, and 3 years after the implantation procedure. Following the intravenous injection of Iodixanol (China, Mainland), CT angiography was provided in the arterial phase and the images were reconstructed by a multisegment algorithm.

\section{Statistical Analysis}

Statistical analysis was performed by independent sample $t$ test of SPSS, version 19 (IBM, SPSS Statistics, IBM Corp, Armonk, NY). The level of significance was considered as $P<.05$.

\section{RESULTS}

All the animals survived through the follow-up period. Eight surgeries were successfully carried out, the scaffolds were grafted to New Zealand rabbits' superficial femoral artery, and the grafted conduits showed no signs of stricture or occlusion in evaluations by Doppler ultrasonography 2 weeks and a month after the surgery. After 3 years of follow-up, the animals were killed and the grafted tissues were explanted for further analysis. The native superficial femoral arteries from animals' opposite extremity were also harvested to be evaluated as a control group (Figure 2).

\section{Scanning Electron Microscopy}

SEM studies on native and acellular IMA confirmed the preservation of the ECM ultrastructure after the decellularization process as no distortion could be detected in ECM bundles in acellular IMA, and microscopic properties of the scaffolds appeared similar to the native IMA. Also, cellular components that were distinguished in the native IMAs as cobblestone-resembling components could not be detected in the acellular IMA. These findings could confirm the decellularization process as well as preservation of the ECM. These data are also supported by histologic and immunohistochemical evaluations (Figure 3).

\section{Histologic and Immunohistochemical Evaluation}

H\&E staining of the native IMA showed endothelial cells and smooth muscle cells in intima and media layers. Histologic evaluation of acellular IMA by H\&E demonstrated the absence of nuclei indicative for complete tissue decellularization. No smooth muscle cells or endothelial cells could be detected in these specimens. Also, ECM structure was maintained in acellular samples, and no deformity or distortion could be detected after the decellularization process. Histologic evaluation of the native IMA by Movat's pentachrome depicted scattered smooth muscle cell lining in media layer and an abundance of collagen and elastic fibers in intima and media layers. Pentachrome staining of acellular IMA samples showed a vast network of collagen fibers and slightly fewer elastic fibers in intima and media layers without any structural distortion. Also, no muscular fibers could be detected in acellular specimens. Fluorescence microscopy evaluation of native and acellular IMA following the Picrosirius red staining was indicative for sufficient preservation of collagen fibers after the decellularization process (Figure 4).

Histologic evaluations of grafted scaffolds and native superficial femoral arteries were carried out with H\&E and trichrome staining 3 years after the implantation. Moreover, the samples were assessed for $\alpha$-SMA, CD31, and CD34 immunohistochemical markers. H\&E staining of the grafted specimens showed fragments of nerve bundles and portions of well-defined vascular channels. An abundance of nuclei was detected in the intimal lining as well as the media layer indicative for cellular regeneration. Also, vascular channels were patent and no notable findings regarding atherosclerosis or calcification could be observed. Native superficial femoral arteries, as control samples, showed completely similar results. Trichrome staining of the study group highlighted fibroconnective tissue, bundles of muscles, and well-defined vascular channels, and no significant difference was found in the control samples. Immunohistochemical assessments of the implanted IMAs and native arteries for $\alpha$-SMA marker were harmonious and showed diffuse reactivity of smooth muscle cells in the 


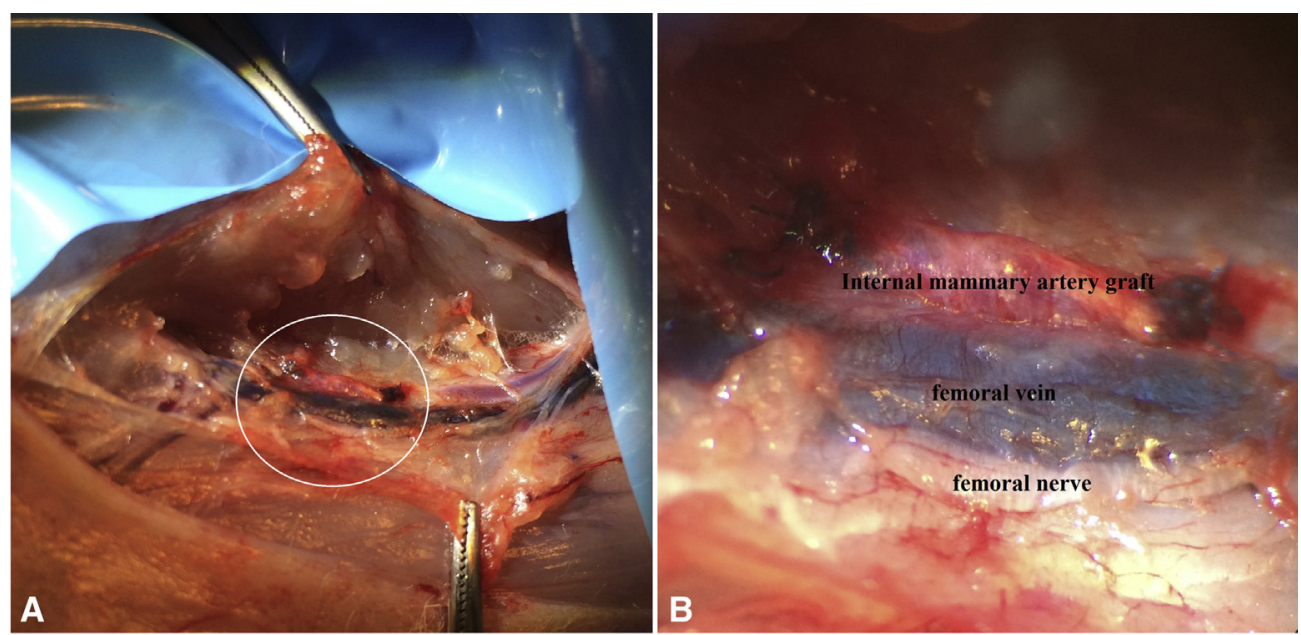

FIGURE 2. Graft explantation. The grafted conduits were harvested after 3 years. Internal mammary arterial graft could be observed adjacent to femoral artery and femoral nerve. The grafted vessel contained normal blood flow. The circle indicates the implanted artery. Both panels A and B demonstrate the grafted acellular internal mammary artery after the follow up period. Panel B is in higher magnification to highlight the graft site and its adjacent nerve and vein.

media layer $(P<.05)$. Furthermore, CD31 and CD34 immunohistochemical staining of both samples manifested positive reactivity of endothelial cells in intima layer $(P<.05)$. Overall, the grafted acellular scaffolds revealed convincing signs of regeneration in all layers and did not show any notable difference to animals' native arteries (Figure 5).

\section{CT Angiography}

Pulse oximetry of the lower limbs showed a peripheral capillary oxygen saturation of $95 \%$ or greater in all animals at the times of follow-up indicative for efficient blood supply and oxygenation. CT angiography evaluations of the vascular network 3 months, 1 year, and 3 years after the

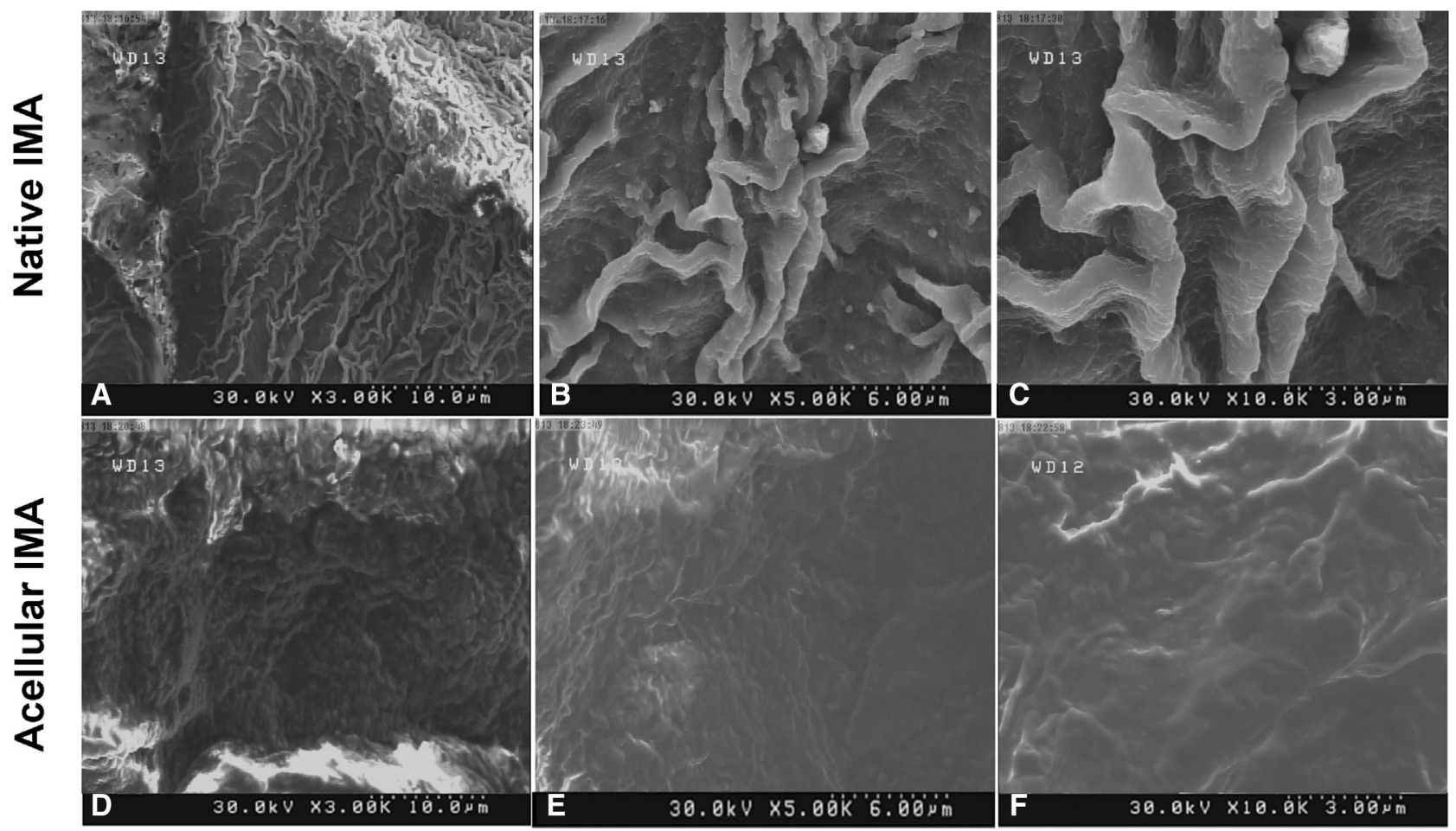

FIGURE 3. Scanning electron microscopy evaluations. Cobblestone-resembling structures that are present in the native IMA (A-C) could not be detected in acellular samples (D-F). Also. the ECM ultrastructure is preserved after the decellularization process. These findings could confirm the decellularization process as well as preservation of the ECM. IMA, Internal mammary artery. 


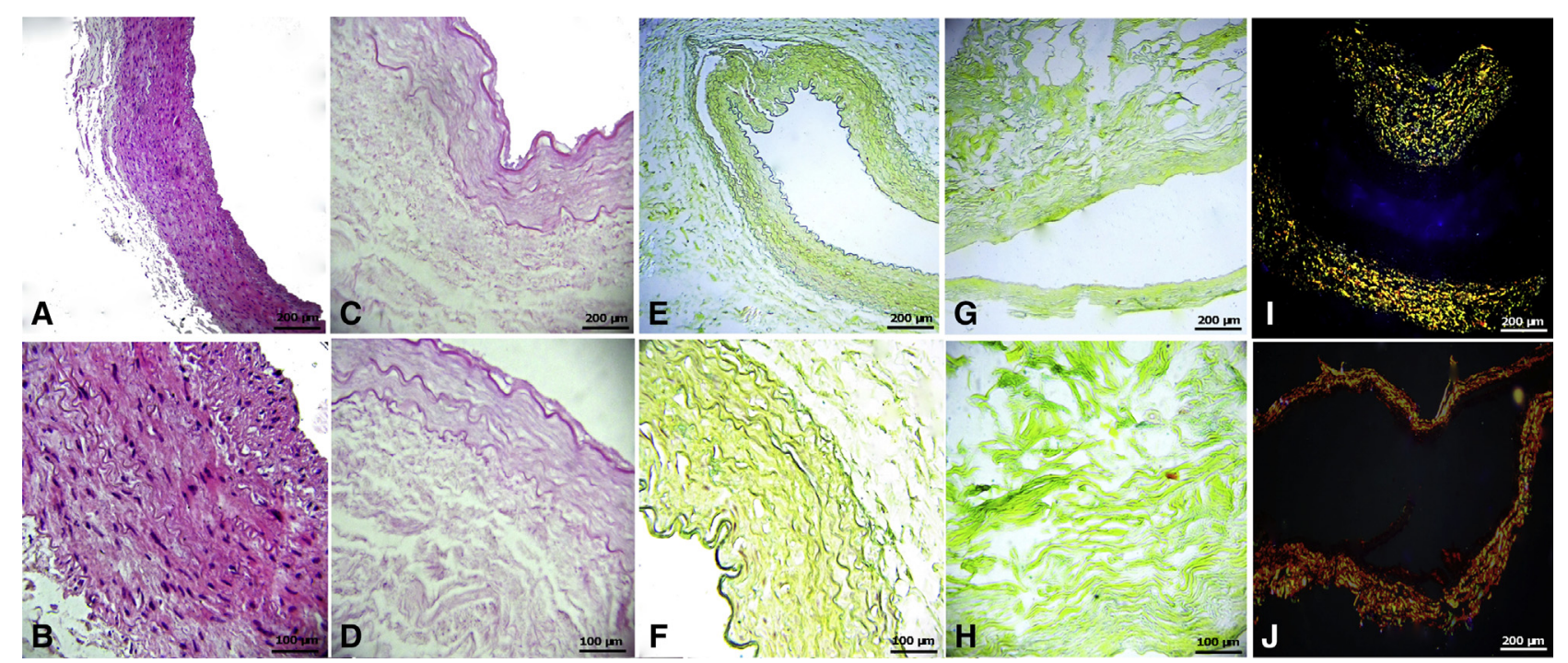

FIGURE 4. A and B, Histologic evaluation of the native IMA by H\&E staining shows endothelial cells and smooth muscle cells in intima and media layers. $\mathrm{C}$ and $\mathrm{D}, \mathrm{H} \& \mathrm{E}$ staining of the acellular IMA depicts the absence of nuclear components indicative for complete tissue decellularization. Also, the ECM structure is maintained in these samples. E and F, Movat's pentachrome staining of the native IMA shows scattered smooth muscle cell lining in media layer and an abundance of collagen and elastic fibers in intima and media layers. G and H, Pentachrome staining of acellular IMA samples showed a vast network of collagen fibers and slightly fewer elastic fibers in intima and media layers without any structural distortion. Also, no muscular fibers could be detected in acellular specimens. Fluorescence microscopy evaluation of native (I) and acellular IMA (J) after the Picrosirius red staining are indicative for sufficient preservation of collagen fibers after the decellularization process.

implantation surgery confirmed patency of the conduits. Both right and left common femoral arteries could be discerned; on the left side (nonimplanted), bifurcation of the common femoral artery could be observed, and on the right side (implanted) the site of the anastomosis could be detected distal to the bifurcation point on the right superficial femoral artery. The absence of vascular cut-off or stricture on the implanted side is a notable finding that could be interpreted as patency of the vascular graft and absence of thrombosis. Also, no evidence of neovascularization could be detected adjacent to the grafted area that confirms efficient blood supply and absence of ischemia in the grafted limbs. To summarize, CT angiographic evaluations confirmed the patency of the grafts and its distal branches in all of the samples without any signs of aneurysm formation or thrombosis at 3 months, 1 year, and 3 years after the implantation (Figure 6).

\section{DISCUSSION}

Currently, autologous saphenous veins are the most commonly used vascular substitutes for coronary arteries because of their ease of access and fewer complications related to their excision; nevertheless, various conduits with greater patency and more functional and mechanical similarities to coronary arteries are being investigated. ${ }^{19}$ Various investigations have considered IMA as the gold standard vascular graft for CABG surgery due to its several favorable characteristics, such as its high patency rate. ${ }^{7,8}$
In the present study, we decellularized human IMAs according to the previously described protocol. ${ }^{17}$ Histologic evaluation of the IMAs before and after the decellularization process confirmed complete cell removal and preservation of the ECM. Also, SEM assessments showed no evidence of ECM ultrastructure distortion after decellularization, which is necessary for maintaining lumen patency. ${ }^{20}$ Furthermore, we implanted the acellular scaffolds into the femoral arteries of 8 New Zealand rabbits. Primarily, rat abdominal aorta and rabbit abdominal aorta were also considered as graft sites; however, these surgeries were terminated after 2 operations for each due to high mortality rates that were caused by long ischemic time of the abdominal aorta and complications related to arterial thrombosis during the time of surgery. The purpose of this study was to evaluate the acellular IMA's patency and its regenerative potential; thus, we continued the surgeries as described for rabbit femoral artery. All of the animals survived through the whole follow-up time. Doppler ultrasonographic evaluation of the grafted conduits confirmed the patency of the scaffolds 2 weeks and a month following the surgery. Also, CT angiographic assessments after 3 months, 1 year, and 3 years revealed complete patency of the lumens without any evidence of stricture or aneurysm formation.

The results of this study, in addition to our previous investigations, ${ }^{17,18}$ provide proof of concept for the application of acellular IMA grafts as efficient vascular substitutes with no-antigenicity, which makes them applicable for 


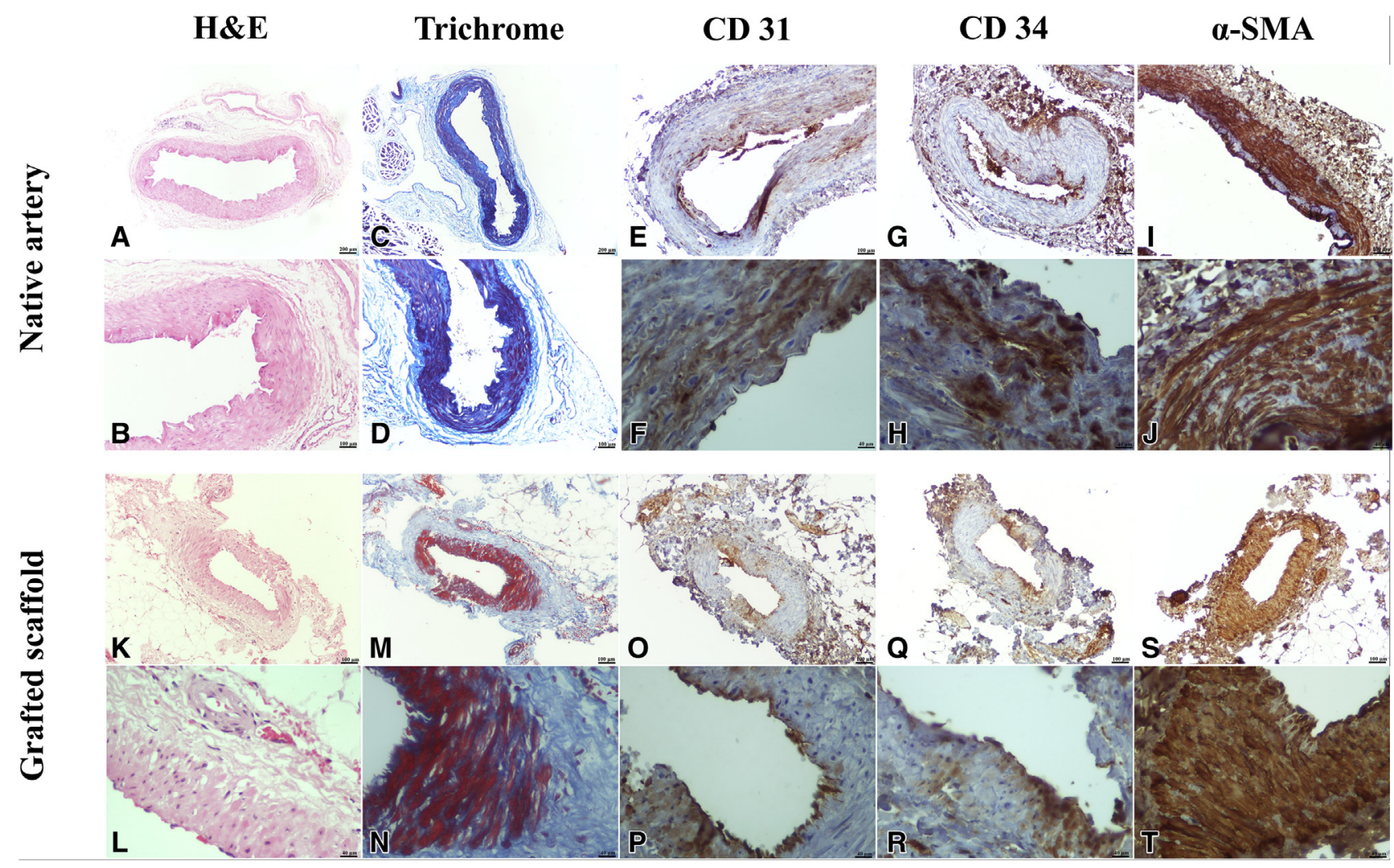

FIGURE 5. (Top) Histologic and immunohistochemical evaluation of the native artery. A and B, Evaluation of native superficial femoral arteries by H\&E staining shows fragments of nerve bundles and portions of well-defined vascular channels. C and D, Trichrome staining of the study group demonstrates well defined vascular channels with fibro-connective tissue and bundles of muscles. E and F, CD31 and (G and H) CD34 staining were positive indicative for reactivity of endothelial cells in intima layer. I and J, Immunohistochemical assessment of the native artery for $\alpha$-SMA marker shows diffuse reactivity. (Bottom) Histologic and immunohistochemical evaluation of the grafted IMA after 3 years. K and L, H\&E staining of the grafted specimens shows well-defined vascular channels. An abundance of nuclei was detected in the intimal lining as well as the media layer indicative for cellular regeneration. No sign of atherosclerosis is detectable. M and N, Evaluation of the study group by trichrome staining demonstrates fibro-connective tissue, bundles of muscles, and well-defined vascular channels. O and P, CD31 and (Q and R) CD34 immunohistochemical staining manifested positive reactivity of endothelial cells in intima layer. S and T, $\alpha$-SMA staining shows diffuse reactivity of smooth muscle cells in the media layer. $H \& E$, Hematoxylin and eosin; $\alpha-S M A$, alpha smooth muscle actin.

allotransplantations. Although arterial grafts retain mechanical and functional similarity to coronary arteries, their application is hampered by vasospasm-related occlusion and vascular stenosis. ${ }^{21}$ Certain studies suggested that the advantages of the application of IMA in comparison with other arterial grafts are related to its fewer smooth muscle cells, vast elastic fibers network, and compacted internal elastic layer restricting the intimal hyperplasia process and consequently the vascular occlusion. ${ }^{22}$ The results of the present study demonstrate that these properties are related to ECM components of the IMA and that the acellular IMA includes the same desirable characteristics without the risk of immune reactions in allo/xeno-grafts. These findings could provide us with an extended source of vascular substitutes for arterial transplantation surgeries, especially when autotransplantation is not a preference due to certain conditions such as systemic vascular diseases.
In a study by Kaushal and colleagues, ${ }^{23}$ preseeded acellular porcine iliac vessels remained patent for 130 days in sheep carotid artery whereas nonseeded grafts were only patent for 15 days. In contrast, in the present investigation, acellular IMA grafts showed no signs of thrombosis or stricture on Doppler ultrasonography and CT angiography evaluations after 3 years.

Furthermore, Conklin and colleagues ${ }^{24}$ performed the carotid artery bypass surgery in canine models with the application of acellular porcine common carotid arteries. Evaluation of the grafted vessels after 2 months demonstrated the recellularization of both media and intima layers. The outcomes of this study are compatible with our findings since the grafted IMAs showed no significant difference compared with animal's native artery in histopathologic and immunohistochemical aspects. Moreover, Tillman and colleagues ${ }^{25}$ investigated the application of porcine 

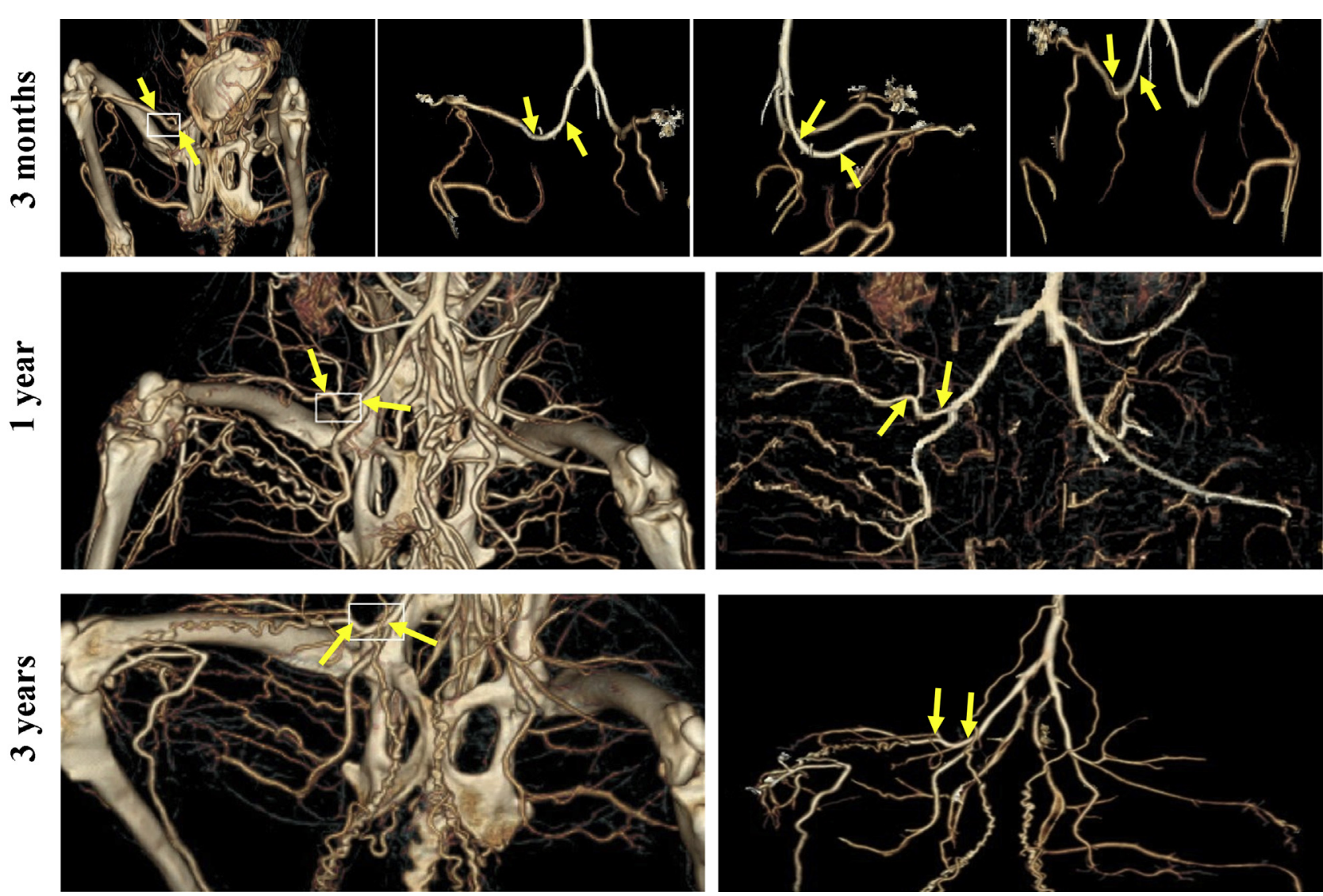

FIGURE 6. CT angiography evaluations of the vascular network (The arrows and the squares indicate the graft site). The grafts and its distal branches were patent during the follow-up time in all of the samples without any signs of aneurysm formation or thrombosis at 3 months, 1 year, and 3 years after the implantation.

tissue-engineered arteries to create AV grafts in ovine models. The average patency rate of the grafts was 4.5 months, and all of the conduits developed venous stenosis at the end of the study.

To the best of our knowledge, this is the first study to investigate the application of acellular IMA as a smalldiameter vascular graft in animal models. The results of this investigation after 3 years of follow-up could pave the way for the future clinical application of this tissueengineered vascular graft. Nevertheless, further studies are required to prove the efficacy of this graft in larger animal models. Absence of evaluating the arterial blood flow at implant and harvest times are limitations to this investigation; however, we examined the blood supply of the organ and the patency of the graft by pulse oximetry and CT angiography. Also, lack of comparison with native IMA and other biomaterials as well as absence of biomechanical studies could be limitations of this study. Future studies should focus on comparing the patency rates of acellular IMA and other vascular grafts in a larger group of animals. We did not perform extended evaluations on the acellular tissue before grafting since this study is based on our previous investigation concerning the decellularization protocol and in vivo recellularization of the IMA. ${ }^{17,18}$ This investigation provides proof of concept that IMA scaffolds could be used as arterial grafts for different small-diameter bypass surgeries in future in vivo or clinical studies. We performed the surgeries on rabbit superficial femoral artery, which approximates the diameter of human small vessels such as coronary arteries, to assess the patency of this conduit as a small-diameter vascular substitute. Nevertheless, further studies are required to examine the efficacy of this graft in CABG surgeries. Evaluation of the acellular IMA as a coronary bypass graft in a large animal model could be the next step of this study.

\section{CONCLUSIONS}

In conclusion, this study provides proof of concept of acellular IMA for the long-term application of acellular IMA as a small-diameter vascular substitute. The grafted scaffolds stayed patent after 3 years of follow-up in all of the animals. All 3 layers of the arterial grafts were recellularized and showed no significant difference compared with the hosts' native arteries. These findings could be used in 
future clinical studies on application of this novel conduit for small-diameter bypass surgeries, including CABG.

\section{Conflict of Interest Statement}

Authors have nothing to disclose with regard to commercial support.

We would like to express our gratitude to Ms Hasti Mahdavi for preparing the preliminary draft of this study.

\section{References}

1. Benjamin EJ, Blaha MJ, Chiuve SE, Cushman M, Das SR, Deo R, et al. Heart Disease and Stroke Statistics_-2017 Update: a report from the American Heart Association. Circulation. 2017:135:e146-603.

2. Quillard T, Franck G, Mawson T, Folco E, Libby P. Mechanisms of erosion of atherosclerotic plaques. Curr Opin Lipidol. 2017;28:434-41.

3. Hlatky MA, Boothroyd DB, Bravata DM, Boersma E, Booth J, Brooks MM, et al. Coronary artery bypass surgery compared with percutaneous coronary interventions for multivessel disease: a collaborative analysis of individual patient data from ten randomised trials. Lancet. 2009:373:1190-7.

4. Hlatky MA, Boothroyd DB, Melsop KA, Brooks MM, Mark DB, Pitt B, et al. Medical costs and quality of life 10 to 12 years after randomization to angioplasty or bypass surgery for multivessel coronary artery disease. Circulation. 2004;110: 1960-6.

5. Campeau L, Enjalbert M, Lespérance J, Bourassa MG, Kwiterovich P Jr, Wacholder S, et al. The relation of risk factors to the development of atherosclerosis in saphenous-vein bypass grafts and the progression of disease in the native circulation. A study 10 years after aortocoronary bypass surgery. N Engl J Med 1984:311:1329-32.

6. Loop FD. Coronary artery surgery. Ann Thorac Surg. 2005;79:S2221-7.

7. Loop FD, Lytle BW, Cosgrove DM, Stewart RW, Goormastic M, Williams GW, et al. Influence of the internal-mammary-artery graft on 10-year survival and other cardiac events. $N$ Engl J Med. 1986;314:1-6.

8. Bello SO, Peng EW, Sarkar PK. Conduits for coronary artery bypass surgery: the quest for second best. J Cardiovasc Med (Hagerstown). 2011:12:411-21.

9. Klinkert P, Post P, Breslau P, van Bockel J. Saphenous vein versus PTFE for above-knee femoropopliteal bypass. A review of the literature. Eur J Vasc Endovasc Surg. 2004;27:357-62.

10. Kappetein AP, van Mieghem NM, Head SJ. Revascularization options: coronary artery bypass surgery and percutaneous coronary intervention. Heart Failure Clin. 2016;12:135-9.

11. Weinberg CB, Bell E. A blood vessel model constructed from collagen and cultured vascular cells. Science. 1986;231:397-400.
12. Pawlowski KJ, Rittgers SE, Schmidt SP, Bowlin GL. Endothelial cell seeding of polymeric vascular grafts. Front Biosci. 2004;9:1412-21.

13. Kameli M, Khorramirouz R, Eftekharzadeh S, Fendereski K, Daryabari SS, Tavangar SM, et al. Application of tissue-engineered pericardial patch in rat models of myocardial infarction. J Biomed Mater Res A. 2018;106: 2670-8.

14. Bader A, Steinhoff G, Strobl K, Schilling T, Brandes G, Mertsching H, et al. Engineering of human vascular aortic tissue based on a xenogeneic starter matrix. Transplantation. 2000; 170:7-14.

15. Assmann A, Delfs C, Munakata H, Schiffer F, Horstkötter K, Huynh K, et al. Acceleration of autologous in vivo recellularization of decellularized aortic conduits by fibronectin surface coating. Biomaterials. 2013;34:6015-26.

16. Dahl SL, Kypson AP, Lawson JH, Blum JL, Strader JT, Li Y, et al Readily available tissue-engineered vascular grafts. Sci Transl Med 2011;3:68ra9.

17. Kajbafzadeh AM, Khorramirouz R, Kameli SM, Hashemi J, Bagheri A. Decellularization of human internal mammary artery: biomechanical properties and his topathological evaluation. Biores Open Access. 2017;6:74-84.

18. Khorramirouz R, Kameli SM, Eftekharzadeh S, Kajbafzadeh AM. Application of omentum as an in vivo bioreactor for regeneration of decellularized human internal mammary artery. J Biomed Mater Res A. 2017;105:2685-93.

19. Pashneh-Tala S, MacNeil S, Claeyssens F. The tissue-engineered vascular graftpast, present, and future. Tissue Eng Part B Rev. October 8, 2015 [Epub ahead of print].

20. Bogorad MI, DeStefano J, Wong AD, Searson PC. Tissue-engineered 3D microvessel and capillary network models for the study of vascular phenomena. Microcirculation. 2017;24.

21. Miwa S, Desai N, Koyama T, Chan E, Cohen EA, Fremes SE. Radial artery angiographic string sign: clinical consequences and the role of pharmacologic therapy. Ann Thorac Surg. 2006:81:112-8; discussion 9.

22. Cooper GJ, Underwood MJ, Deverall PB. Arterial and venous conduits for coronary artery bypass. A current review. Eur J Cardiothorac Surg. 1996; 10:129-40.

23. Kaushal S, Amiel GE, Guleserian KJ, Shapira OM, Perry T, Sutherland FW, et al Functional small-diameter neovessels created using endothelial progenitor cells expanded ex vivo. Nat Med. 2001;7:1035-40.

24. Conklin BS, Richter ER, Kreutziger KL, Zhong DS, Chen C. Development and evaluation of a novel decellularized vascular xenograft. Med Eng Phys. 2002;24: 173-83.

25. Tillman BW, Yazdani SK, Neff LP, Corriere MA, Christ GJ, Soker S, et al. Bioengineered vascular access maintains structural integrity in response to arteriovenous flow and repeated needle puncture. J Vasc Surg. 2012;56:783-93.

Key Words: internal mammary artery, vascular graft, tissue engineering, recellularization 